\title{
Magnetic structure of the antiferromagnetic half-Heusler compound NdBiPt
}

\author{
R. A. Müller, ${ }^{1, *}$ A. Desilets-Benoit,,${ }^{1, *}$ N. Gauthier,,${ }^{1} \dagger$ L. Lapointe, ${ }^{1,}{ }^{*}$ A. D. Bianchi, ${ }^{1, \ddagger}$ T. Maris, ${ }^{2}$ R. Zahn, ${ }^{3}$ R. Beyer, ${ }^{3}$ \\ E. Green, ${ }^{3}$ J. Wosnitza, ${ }^{3}$ Z. Yamani, ${ }^{4}$ and M. Kenzelmann ${ }^{5}$ \\ ${ }^{1}$ Département de Physique, Université de Montréal, Montréal, Canada \\ ${ }^{2}$ Département de Chimie, Université de Montréal, Montréal, Canada \\ ${ }^{3}$ Hochfeld-Magnetlabor Dresden (HLD-EMFL), Helmholtz-Zentrum Dresden-Rossendorf, Dresden, Germany \\ ${ }^{4}$ Canadian Neutron Beam Centre, National Research Council, Chalk River, Canada \\ ${ }^{5}$ Laboratory for Scientific Developments and Novel Materials, Paul Scherrer Institut, Villigen, Switzerland
}

(Received 22 June 2015; published 30 November 2015)

\begin{abstract}
We present results of single-crystal neutron-diffraction experiments on the rare-earth, half-Heusler antiferromagnet (AFM) NdBiPt. This compound exhibits an AFM phase transition at $T_{\mathrm{N}}=2.18 \mathrm{~K}$ with an ordered moment of 1.78(9) $\mu_{B}$ per $\mathrm{Nd}$ atom. The magnetic moments are aligned along the [001] direction, arranged in a type-I AFM structure with ferromagnetic planes, alternating antiferromagnetically along a propagation vector $\tau$ of (100). The $R \mathrm{BiPt}(R=\mathrm{Ce}-\mathrm{Lu})$ family of materials has been proposed as candidates for a new family of antiferromagnetic topological insulators (AFTIs) with a magnetic space group that corresponds to a type-II AFM structure where ferromagnetic sheets are stacked along the space diagonal. The resolved structure makes it unlikely that NdBiPt qualifies as an AFTI.
\end{abstract}

DOI: 10.1103/PhysRevB.92.184432

PACS number(s): 75.25.-j, 75.50.Ee, 73.20.-r

\section{INTRODUCTION}

A usual concept in physics is the occurrence of some form of symmetry breaking at phase transitions between different states of matter. In 1980, Klaus von Klitzing widened that concept by describing a new quantum state of matter which does not follow this pattern, but sheds light on a new family of materials, only characterized by their Hilbertspace topology. In this new state of matter, the bulk of a two-dimensional sample stays insulating, whereas along its edges a unidirectional current is circulating, giving rise to the quantum Hall effect in a two-dimensional electron gas (2DEG). Inspired by the mathematical field of topology, the quantized conductivity of such a material can be associated with a topological invariant. In mathematics, such an invariant describes a property of a topology that remains unchanged under homeomorphisms. For example, the number of holes in a two-dimensional manifold cannot be changed by stretching it.

In solid-state physics, we can adapt this concept of smooth deformations to the topology of the Hilbert space, which describes the band structure of an insulator. As long as these transformations are adiabatic, the topological invariant will not change, and, therefore, the band gap at the Fermi level of the material remains unaffected. While the quantum Hall state in a 2DEG requires an applied magnetic field, in the case of a $\mathrm{Hg} / \mathrm{CdTe}$ quantum well, strong spin-orbit coupling acts as an effective field [1]. If the well is thinner than a critical value $d_{c}$, it behaves like a conventional insulator. For $d_{Q W}>d_{c}$, the topological invariant changes and a single pair of helical edge stages that form a Kramers pair counterpropagate on the same

\footnotetext{
*Regroupement Québécois sur les Matériaux de Pointe (RQMP).

${ }^{\dagger}$ Regroupement Québécois sur les Matériaux de Pointe (RQMP); Current address: Laboratory for Scientific Developments and Novel Materials, Paul Paul Scherrer Institut, Villigen, Switzerland.

${ }^{\ddagger}$ Regroupement Québécois sur les Matériaux de Pointe (RQMP); andrea.bianchi@umontreal.ca
}

edge. In consequence, the magnetotransport in such quantum wells shows steps [2].

Spin-orbit coupling is also at the origin of topological insulators in three dimensions [3-5]. Experimentally, spinand angle-resolved photoemission spectroscopy on bismuth doped with antimony showed the presence of metallic surface states, as well as a spin texture [6].At the same time, $a b$ initio calculations predicted a small gap in the electronic spectrum for the bulk of this material [7].

Recently, theorists have brought forward several propositions suggesting that half-Heusler compounds, showing antiferromagnetic order, could host a new class of topological insulators. Mong et al. [8] described a new symmetry class, where both time-reversal and lattice translational symmetry of an antiferromagnet are broken, yet their product is preserved, resulting in a new antiferromagnetic topological insulator (AFTI) phase. The broken time-reversal symmetry of AFTI is what distinguishes them from conventional topological insulators, where the time-reversal symmetry has to be present for the surface states to occur, which forbids magnetic order. Described in their paper as model B [8] the orientation of the magnetic moment can introduce a net magnetization between intermediate nonmagnetic sites creating an Aharonov-Bohmlike flux which acts as Rashba spin-orbit coupling, resulting in a nontrivial topological phase.

Heusler and the derivative half-Heusler materials can be characterized as semimetals displaying insulating or semimetallic behavior in electrical transport measurements. This behavior agrees with band-structure calculations, which for many of these compounds show a single band crossing the Fermi surface, which led to the proposition that conventional topological insulators can be found in this class of compounds [5,9-11]. $R \mathrm{BiPt}$ materials, where $R$ is a rare-earth metal, first reported in detail in 1991 [12], display a whole set of emergent behaviors ranging from a massive electron state in YbBiPt [13] to superconductivity without inversion symmetry in LaBiPt [14], LuBiPt [15], and YBiPt [16-18], to $\mathrm{CeBiPt}$ which shows a magnetic-field-induced change of 
the Fermi surface [19]. This also prompted investigations of the $R \mathrm{BiPd}$ [20] versions which led to the discovery of superconducting LuBiPd, which shows an anomaly in the electronic specific heat at the superconducting transition, and weak antilocalization in the magnetic-field dependence of the electrical resistivity, which is characteristic for 2D conduction [21].

Angle-resolved photoemission spectroscopy (ARPES) experiments on $\mathrm{Lu}$, Dy, and GdBiPt have shown indications of metallic surface states that differ from the bulk band structure. Liu et al. [22] found that within their resolution an even number of bands cross at the chemical potential, making surface states vulnerable to nonmagnetic backscattering, and these materials should, therefore, not be qualified as strong topological insulators. An inelastic x ray [23] as well as a powder neutron diffraction experiment [24] on GdBiPt indicate a doubling of the unit cell along its space diagonal with the moments arranged in ferromagnetic sheets [24] normal to the [111] direction, leading to a path asymmetry for hopping between nonmagnetic sites, as proposed by Mong et al., and, therefore, making this material a strong candidate for the AFTI phase.

This has prompted us to carry out single-crystal neutron and $\mathrm{x}$-ray diffraction, as well as thermodynamic and transport experiments, to determine the magnetic structure of NdBiPt, because its crystalline structure has all the necessary symmetries for being an AFTI.

\section{SAMPLES AND EXPERIMENT}

$\mathrm{NdBiPt}$ was grown using $\mathrm{Bi}$ flux. $\mathrm{Nd}, \mathrm{Bi}$, and $\mathrm{Pt}$ of high purity were placed in a ceramic crucible in the ratio 1:15:1, which was then sealed in a quartz ampoule under argon atmosphere. The melt was kept at $1200^{\circ} \mathrm{C}$ for two days and then cooled down to $550^{\circ} \mathrm{C}$ over a week, after which the ampoules were taken out of the furnace and centrifuged to separate the flux from the crystals.

Magnetic measurements were taken between 1.8 and $300 \mathrm{~K}$ in an applied field of $0.1 \mathrm{~T}$ using a Quantum Design VSM SQUID magnetometer. Resistivity was measured in the same temperature range with a Quantum Design PPMS using four-point contacts. The specific heat $C_{p}$ was measured in a ${ }^{3} \mathrm{He}$ insert PPMS using a standard puck but purpose-built electronics.

Single-crystal x-ray diffraction data were collected at $150 \mathrm{~K}$ on a Bruker D8 VENTURE diffractometer with a CMOS PHOTON 100 detector and a liquid-metal jet $\mathrm{x}$-ray source using Ga radiation $(\lambda=1.3414 \AA)$. The data set was collected using a combination of $\omega$ and $\phi$ scans with a step size of $1^{\circ}$, and $1 \mathrm{~s}$ exposure per frame. Data collection and unit-cell lattice parameters determination were performed with the APEX2 suite [25]. Final lattice-parameter values and integrated intensities were obtained using SAINT software, and a multiscan absorption correction was applied with SADABS [26]. The structure was refined with SHELXL version 2014/3 [27].

For the single-crystal neutron-diffraction experiment we co-aligned three crystals of the size of the order $2 \times 1 \times 1 \mathrm{~mm}^{3}$ on an aluminum plate. We oriented our crystals to be able to scan the $(h h l)$ scattering plane given the extinction rules of the $\mathrm{NdBiPt}$ crystalline structure. Also, this scattering geometry allowed us to distinguish between the type-I AFM order, seen in the isostructural CeBiPt [29], and type-II AFM order, as proposed by Mong et al. in Ref. [8] and observed in GdBiPt [24]. The experiment was carried out on the C5 triple-axis spectrometer at the Canadian Neutron Beam Centre in Chalk River. A vertically focusing pyrolytic graphite (PG) (002) monochromator and a flat $\mathrm{PG}(002)$ analyzer crystal were used with a fixed final neutron energy of $E_{f}=14.56 \mathrm{meV}$, with no collimation, and collimations of $0.8^{\circ}, 0.85^{\circ}$, and $2.4^{\circ}$. Two PG filters were placed in the diffracted beam after the sample to eliminate higher-order wavelength contamination of the beam. The sample was sealed under helium gas in an aluminum can and mounted in a closed-cycle ${ }^{3} \mathrm{He}$ Heliox Displex cryostat that allowed cooling the sample down to $0.3 \mathrm{~K}$.

\section{RESULTS AND ANALYSIS}

\section{A. X-ray diffraction}

NdBiPt crystallizes in the cubic half-Heusler crystal structure with the space group $F \overline{4} 3 m$. [12] This structure consists of four interpenetrating $f c c$ lattices shifted by $\left[\frac{1}{4}, \frac{1}{4}, \frac{1}{4}\right]$, where the $\left[\frac{1}{2}, \frac{1}{2}, \frac{1}{2}\right]$ position is an ordered vacancy. The refinement of our single-crystal $\mathrm{x}$-ray patterns confirms this structure (for details see Tables I and II in the Supplemental Material [28]). The compound has a lattice constant of 6.7613(2) $\AA$ with the $\mathrm{Nd}^{3+}$ ion located on the $\left[\frac{1}{4}, \frac{1}{4}, \frac{1}{4}\right](4 \mathrm{c})$, Bi on the $\left[\frac{3}{4}, \frac{3}{4}, \frac{3}{4}\right](4 \mathrm{~d})$, and $\mathrm{Pt}$ on the $[0,0,0]$ (4a) position, and permutations of $\left[0, \frac{1}{2}, \frac{1}{2}\right]$ (corresponding to column D of Table II of the Supplemental Material).

In a noncentrosymmetric structure, anomalous x-ray scattering leads to different intensities for so-called Friedel pairs, such as $(h k l)$ and $(\bar{h} \bar{k} \bar{l})$. The refinement confirms the original structure (see Fig. 1 of the Supplemental Material), resulting in an $R 1$ value of 0.0582 , where $R 1$ is the difference between the experimental observed squares of the structure factors for all

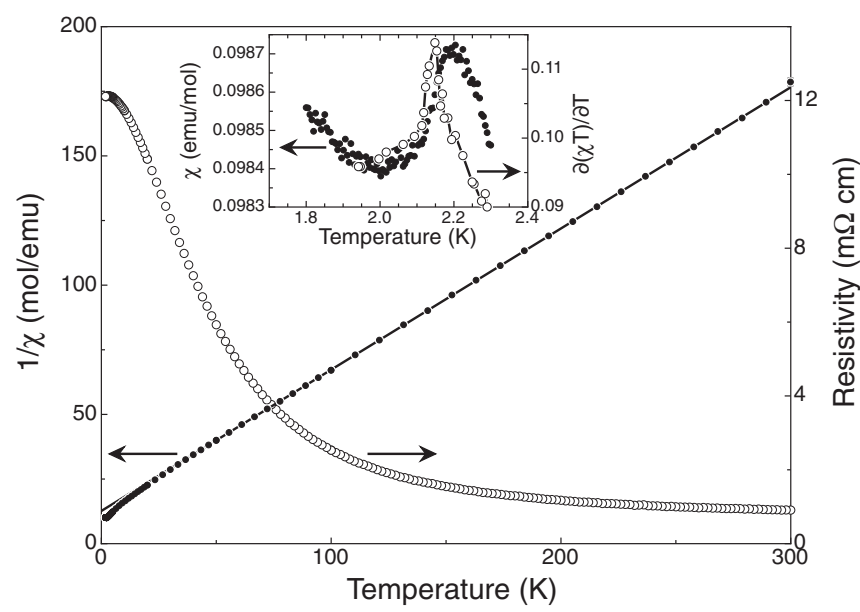

FIG. 1. Inverse magnetic susceptibility measured at $0.1 \mathrm{~T}$ and resistivity (at $0 \mathrm{~T}$ ) as a function of temperature. The inverse susceptibility has been fitted with a Curie-Weiss law in the hightemperature regime yielding $\Theta_{\mathrm{W}}=-23 \mathrm{~K}$ with an effective moment of $\mu_{\text {eff }}=3.73 \mu_{\mathrm{B}}$. Inset: Temperature derivative $\partial(T \chi) / \partial T$ of the magnetic susceptibility showing a sharp peak at the critical temperature $T_{\mathrm{N}}$ of $2.18 \mathrm{~K}$. 
observed peaks, and the respective calculated values. Also the Flack parameter for the original structure is $0.28(3)$, which is the absolute structure factor. This is in contrast to a $R 1$ value of 0.0800 and Flack parameter of 0.72(4) for the inverted structure, as listed in Table II of the Supplemental Material. Please note that a Flack parameter should be close to 0 for a correct structure and close to 1 for an inverted structure.

\section{B. Magnetic and transport properties}

NdBiPt is a semimetal with a very low charge-carrier density, and a high charge-carrier mobility [30] For the temperature range $50-300 \mathrm{~K}$, the magnetic susceptibility $\chi=$ $\frac{M}{H}$ measured in an applied field of $0.1 \mathrm{~T}$ shows a Curie-Weiss behavior with a Curie-Weiss temperature $\Theta_{\mathrm{W}}$ of $-23 \mathrm{~K}$ (see Fig. 1), and an effective magnetic moment $\mu_{\text {eff }}$ of $3.73 \mu_{\mathrm{B}}$ consistent with the theoretical value of $3.62 \mu_{\mathrm{B}}$ for a free $\mathrm{Nd}^{3+}$ ion. The inset of Fig. 1 shows $\chi(T)$ in the temperature range between 1.8 and $2.4 \mathrm{~K}$, where the main features are a maximum at $2.2 \mathrm{~K}$ and a subsequent point of inflection at $2.18 \mathrm{~K}$, confirming antiferromagnetic order with a Néel temperature $T_{\mathrm{N}}$ of $2.18 \mathrm{~K}$ [31]. All three measurements, i.e., specific heat $C_{p}$ (see Fig. 5), electrical resistivity $\partial \rho / \partial T$ (not shown), and magnetic susceptibility $\partial(T \chi) / \partial T$ (inset of Fig. 1), show a discontinuity at the same critical temperature $T_{\mathrm{N}}$, giving evidence for the high quality of our samples.

\section{Neutron diffraction}

Neutron-diffraction data were collected between 0.3 and $5 \mathrm{~K}$. We used a linear fit for the background. Our measurements show a slight mosaic due to a small misalignment of the three crystals of about one degree, as can be seen in the peak shape in Fig. 2(a). To correct for the mosaic the peaks were fitted with a double Gaussian:

$$
\begin{aligned}
G(x)= & B+A \exp \frac{-4(\ln 2)\left|x-x_{0}\right|^{2}}{s^{2}} \\
& \times\left\{1+\frac{1}{R} \exp \frac{4(\ln 2)\left(\Delta^{2}+2\left|x-x_{0}\right| \Delta\right)}{s^{2}}\right\},
\end{aligned}
$$

where $B$ corrects for an imperfect background subtraction. $A$ is the amplitude and $x_{0}$ denotes the center position of the dominant peak. The parameter $s$ represents the full width at half maximum (FWHM), $R$ is the ratio in intensity of the two peaks, and $\Delta$ represents the distance between the two peak centers along $x$.

All the observed magnetic peaks could be indexed as integer fractions of the nuclear peaks which is evidence for a commensurate magnetic structure [see Fig. 3(b)]. For spins located on an $f c c$ lattice, only four types of commensurate antiferromagnetic order are possible [32]. To determine the direction of the magnetic moment, we compare the intensities of the (110) peak with those of the (001) peak. The intensities observed at these two Bragg spots indicate that the magnetic moment is aligned parallel to the momentum of the incoming neutron beam, along the [001] direction, as shown in the inset of Fig. 2(a).

Due to the cubic structure of the crystal, the magnetic moment can point along any of the six edges of the cube, giving rise to three equally probable magnetic domains, which
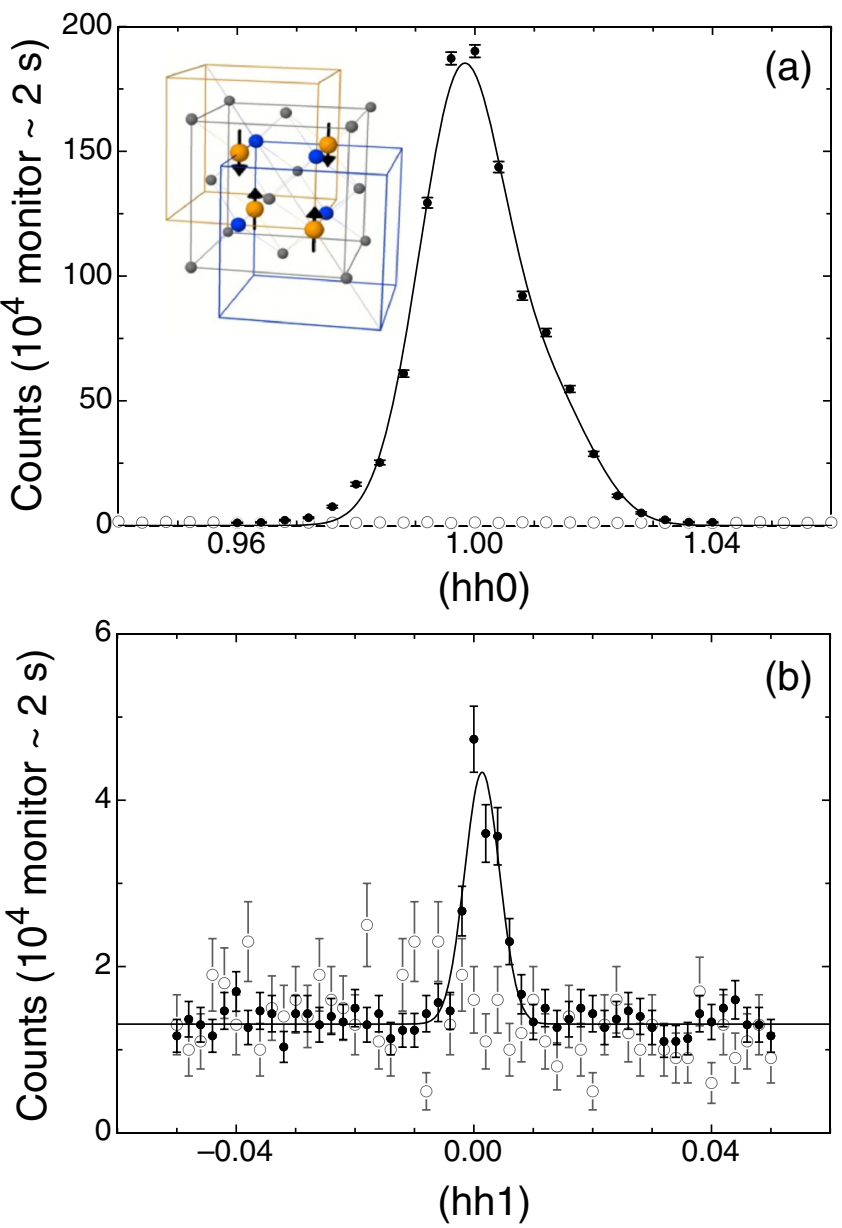

FIG. 2. (Color online) (a) Scan along the $(h h 0)$ direction at $0.3 \mathrm{~K}$ showing the (110) magnetic peak (full circles). The open circles are the signal at $5 \mathrm{~K}$ above $T_{\mathrm{N}}$ (open circles). The inset shows the crystal structure of NdBiPt including the three sublattices for the three different atomic species. (b) Magnetic signal at $0.3 \mathrm{~K}$ (full circles) below $T_{\mathrm{N}}$ due to secondary scattering of neutrons which were first diffracted by the (111) nuclear peak (signal at $5 \mathrm{~K}$ shown as open circles). This position in reciprocal space corresponds to a (001) magnetic peak, though with a much reduced intensity.

are equivalent by symmetry. In our scattering geometry, the structure factor is such that the signals from two of these domains are canceled, leaving only the [001] domain observable. From this, we conclude that the magnetic moment of the $\mathrm{Nd}^{3+}$ ion points normal to the $\{100\}$ family of planes. This means that in $\mathrm{NdBiPt}$ the moment lies along the [100], or the equivalent [010], and [001] directions [inset of Fig. 2(a)]. As we have no reason to assume that one of these domains is preferentially populated, such as can be achieved through the application of mechanical strain to the sample or by applying a magnetic field, we expect all three domains have the same probability to occur. We accounted for the existence of domains when we calculated the size of the ordered magnetic moment. We also would like to point out that these domains are large, since the widths of the magnetic peaks are comparable to the widths of the nuclear peaks which are limited by the instrument and the particular instrument setup we used. In principle, a so-called multi- $\vec{k}$-structure with multiple propagation vectors could also 


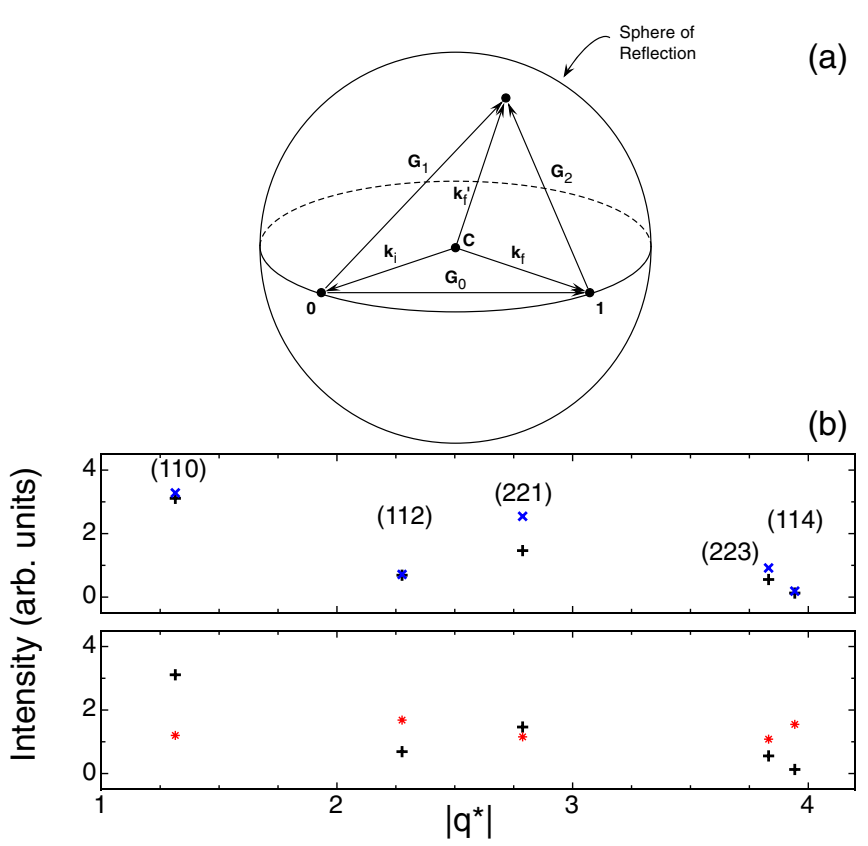

FIG. 3. (Color online) (a) Neutrons diffracted by $\mathbf{G}_{1}$ undergo a second scattering by a reciprocal lattice vector $\mathbf{G}_{2}=\mathbf{G}_{0}-\mathbf{G}_{1}$ [35]. (b) The measured intensities are shown as black crosses. The blue diagonal crosses reflect the refined intensities using FULLPROF with the correct basis (top), and the red stars with the wrong basis set (bottom). For a propagation vector $\tau$ of (100) the correct basis corresponds to magnetic moments which are aligned along the crystallographic $c$ axis.

explain the observed peak intensities; however, we believe this to be unlikely due to the most probable Heisenberg nature of the magnetic interactions in $\mathrm{NdBiPt}$ [33].

The observed structure has ferromagnetic ordered planes with alternating spin direction along the propagation vector $\tau=(100)$, similar to what previously has been observed in the isostructural compound CeBiPt [29]. However, the magnetic order required for the AFTI phase has to have a magnetic-moment component that lies in the Nd plane of the structure, as this would add a net magnetic field which has to be accounted for in the spin-orbit Hamiltonian with an additional Aharonov-Bohm phase that is proportional to the in-plane magnetization [8]. We find that the moments in NdBiPt are aligned perpendicular to the $\mathrm{Nd}$ layer, resulting in a zero net in-plane magnetization, and, therefore, the magnetic order has no impact on the strength of the spin-orbit interaction, because the spins on two neighboring $\mathrm{Nd}$ atoms always cancel each other. We, therefore, conclude that NdBiPt does not qualify as representative of the $S$-symmetry class as described in the article of Mong et al [8]

We performed a single-crystal refinement of the integrated peak intensities using the FULLPROF suite [34]. A representational analysis used BASIREPS for the space group $F \overline{4} 3 m$ with a propagation vector $\tau$ of (001) of this type-I AFM structure, i.e., the decomposition of the magnetic representation in terms of nonzero irreducible representations of all the symmetry groups that leave $\tau$ invariant into the so-called little groups. This analysis results in two sets of basis functions which are listed in Table I.
TABLE I. Real (BASR) and imaginary (BASI) components of the basis vectors for the two permitted commensurable magnetic structures obtained from BASIREPS and the resulting RF factors from the FULLPROF refinement, for the space group $F \overline{4} 3 m$ with an ordering wave vector $\tau$ of [001], and $\mathrm{Nd}^{3+}$ occupying the $4 c$ crystallographic site (see Supplemental Material).

\begin{tabular}{lcccccc}
\hline \hline & Set 1 & RF factor & \multicolumn{2}{c}{ Set 2 } & RF factor \\
\hline BASR & $\left(\begin{array}{lllll}0 & 0 & 1\end{array}\right)$ & 11.5 & $\left(\begin{array}{llll}1 & 0 & 0\end{array}\right)$ & $\left(\begin{array}{llll}0 & 1 & 0\end{array}\right)$ & 47.2 \\
BASI & $\left(\begin{array}{llll}0 & 0 & 0\end{array}\right)$ & & $\left(\begin{array}{llll}0 & 0 & 0\end{array}\right)$ & $\left(\begin{array}{llll}0 & 0 & 0\end{array}\right)$ & \\
\hline
\end{tabular}

The refinement of nuclear peaks followed by the magnetic refinement results in a magnetic moment of $1.78(8) \mu_{\mathrm{B}}$ with an RF factor of 11.5, where the RF factor is the difference between the observed structure factors and the square root of the calculated structure factors. The difference between the two representations is illustrated in Fig. 3(b). It can be seen that the (221) peak shows a higher intensity than the (112) peak in agreement with the experimentally observed intensities, as expected for the magnetic structure presented in the inset of Fig. 2(a). The value for the magnetic moment which we obtain from our refinement is considerably lower than the value of $3.8 \mu_{\mathrm{B}}$ obtained from Curie-Weiss analysis of the high-temperature susceptibility data. This reduction can be accounted for by crystalline electric field effects (CEFs, see Sec. IV).

We did observe a small magnetic signal at the (001) position below the critical temperature, as shown in Fig. 2(b). We can exclude higher harmonics of the fundamental wavelength as the source of this signal due to the presence of PG filters. This led us to the conclusion that the observed intensity must result from second scattering: The incoming beam is first diffracted by the nuclear [111] plane, as schematically shown in Fig. 3(a). The diffracted beam does now allow for a small magnetic intensity at the same position, which would correspond to a (001) magnetic reflection of the primary beam.

An estimate of the strength of a (001) magnetic peak due to secondary scattering can be obtained by using the outgoing flux from the (111) nuclear peak as the incident beam that causes the (001) reflection. This estimate results in an integrated intensity, which is only about $10 \%$ higher than the observed one, thus substantiating our conjecture.

Figure 4(a) shows the temperature dependence of the integrated intensity of the (110) magnetic peak as we cross the transition temperature. To obtain the Néel temperature of $T_{\mathrm{N}}=2.177 \pm 0.005 \mathrm{~K}$, the data were fitted to the scaling law in the temperature range between 1.6 and $2.3 \mathrm{~K}$ [see Fig. 4(a)]:

$$
I=C\left(1-\frac{T}{T_{\mathrm{N}}}\right)^{2 \beta},
$$

yielding a critical exponent of $\beta=0.370 \pm 0.003$, which is close to the value of $\beta=0.369(2)$ expected for a threedimensional Heisenberg antiferromagnet [36]. Figure 4(b) shows the temperature dependence of the Gaussian peak width along the (110) direction, which is proportional to the average inverse correlation length $1 / \xi$. One can see that $\xi$ diverges as we cross the transition temperature, indicating long-range magnetic order. 

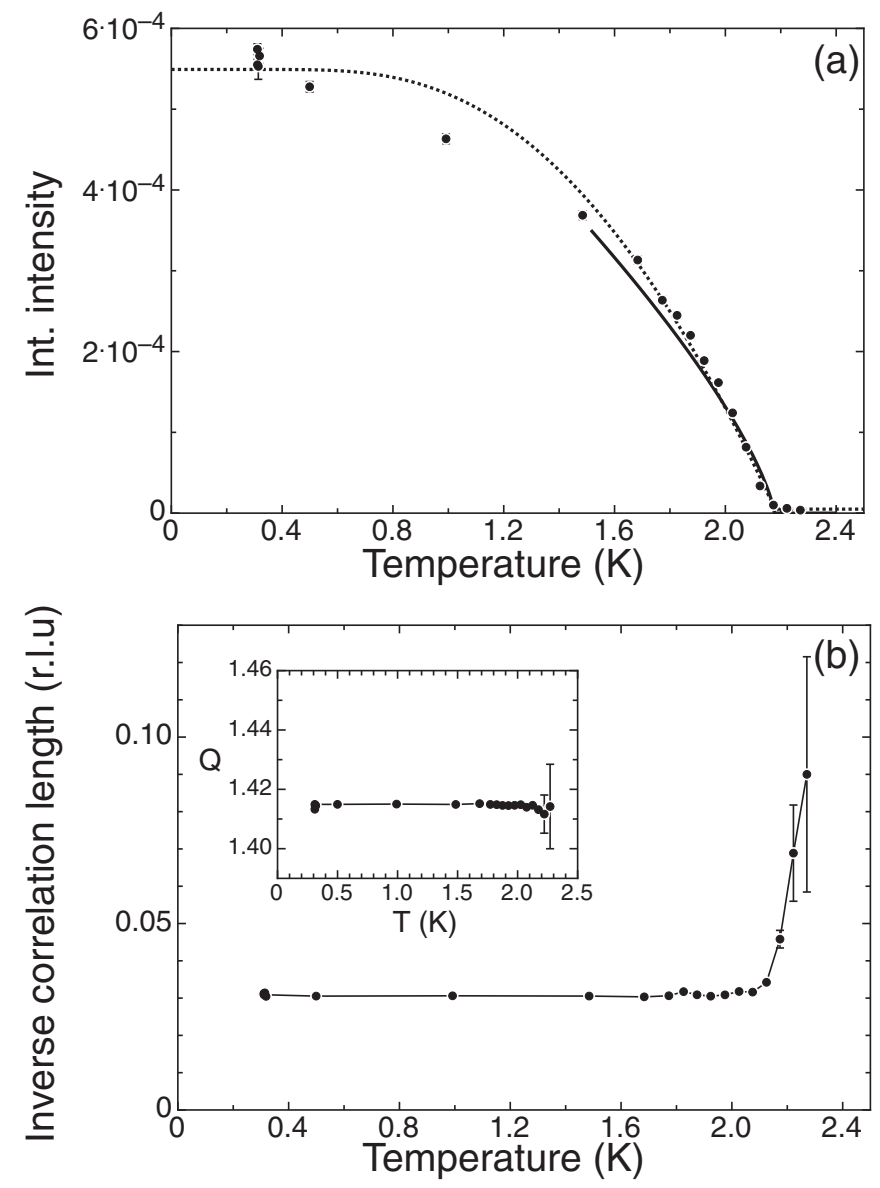

FIG. 4. (a) Temperature dependence of the integrated intensity of the (110) magnetic Bragg reflection. The solid line shows the scalinglaw fit according to Eq. (2) used to determine $T_{\mathrm{N}}$. The dashed line is the fit of the intensity to the Brillouin function of the CEF doublet. (b) Temperature dependence of the inverse correlation length. Inset: Peak position in $q$ space. The solid lines are guides to the eye.

\section{CRYSTALLINE ELECTRIC FIELD EFFECTS}

As noted in Sec. III C, the ordered magnetic moment observed in neutron scattering of $1.78(9) \mu_{\mathrm{B}}$, is strongly reduced compared to the free-ion value of $\mathrm{Nd}^{3+}$ of $3.62 \mu_{\mathrm{B}}$. Since in our scattering geometry only the signal from one of the three domains contributes, we effectively only observed $1 / 3$ of the total magnetic moment in our measurement. Here we assumed that all three domains have an equal probability, since the observed magnetic structure does not break any additional symmetry, besides doubling the unit cell. Such a reduction of the magnetic moment is often observed in intermetallic compounds due to crystalline electric field (CEF) effects. A similar moment reduction to CEF effects was reported for $\mathrm{CeBiPt}$, where the ordered moment corresponds to the magnetic moment of the $\Gamma_{8}$ ground state of the $\mathrm{Ce}^{3+}$ ion [29,37].

The effect of the CEF is to lift the 10-fold degeneracy of the $J=\frac{9}{2}$ multiplet of the $\mathrm{Nd}^{3+}$ ion through an electrostatic interaction. For a $\mathrm{Nd}^{3+}$ ion sitting in a cubic environment, the CEF splitting is expected to result in a new ground state consisting of a $\Gamma_{6}$ doublet and two quartets, $\Gamma_{8}^{(1)}$ and $\Gamma_{8}^{(2)}[38]$.

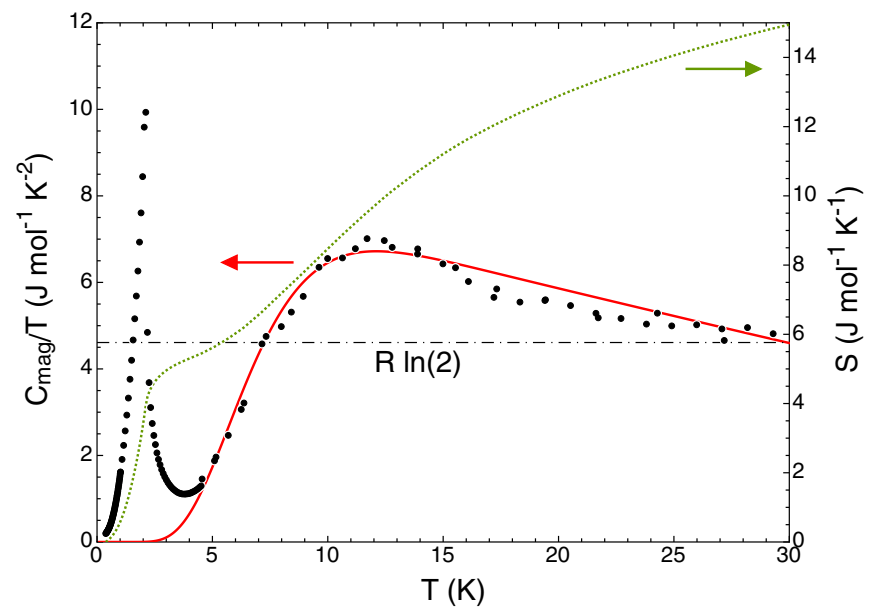

FIG. 5. (Color online) Magnetic contribution to the specific heat $C_{\text {mag }}$ shown as $\frac{C_{\mathrm{mag}}}{T}$ vs $T$. The solid line is the best fit of a Schottky anomaly by using all possible energy eigenvalue configurations obtained by solving the CEF Hamiltonian. The dotted line shows the temperature dependence of the magnetic entropy $S_{\text {mag }}$, which displays a plateau at $R \ln 2$ indicating the $\Gamma_{6}$ doublet as the CEF ground state.

To further investigate the conjecture that this reduction might be due to CEF, we carried out specific-heat measurements in zero field from 0.3 to $30 \mathrm{~K}$. The total specific heat, $C_{p}=C_{\mathrm{el}}+C_{\mathrm{ph}}+C_{\mathrm{mag}}$, is the sum of the electronic contribution $C_{\mathrm{el}}=\gamma T$, the phonon contribution $C_{\mathrm{ph}}$, and the magnetic contribution $C_{\mathrm{mag}}$ we are interested in. Due to the large phonon $C_{\mathrm{ph}}$ and magnetic $C_{\text {mag }}$ contributions in the measured temperature range, we were not able to determine the electronic contribution $C_{\mathrm{el}}$ and could only establish that it is below $1 \mathrm{~mJ} \mathrm{~mol}^{-1} \mathrm{~K}^{-2}$. Such a low value for $C_{\mathrm{el}}$ is expected because of the low charge-carrier concentration in NdBiPt. Over the measured temperature range, $C_{\mathrm{ph}}$ can be described by the Debye function:

$$
C_{\mathrm{ph}}=9 R\left(\frac{T}{\theta_{\mathrm{D}}}\right)^{3} \int_{0}^{\frac{\theta_{\mathrm{D}}}{T}} \frac{x^{4} e^{x}}{\left(e^{x}-1\right)^{2}} d x,
$$

where $R$ is the universal gas constant and $\theta_{\mathrm{D}}$ the Debye temperature. As can be seen in Fig. 5, we observe a rather broad magnetic peak between 4.5 and $20 \mathrm{~K}$, which makes it difficult to fit the phonon contribution. We instead chose to use the $\theta_{\mathrm{D}}$ value of $122.3 \mathrm{~K}$ obtained from a fit of Eq. (3) to the specific-heat data of GdBiPt, which does not have CEF splitting [24]. We then scaled the Debye temperature with the square root of the inverse mass ratio between $\mathrm{Gd}$ and $\mathrm{Nd}$. This yields a Debye temperature of $\theta_{\mathrm{D}}=123.7 \mathrm{~K}$ for NdBiPt. Figure 5 shows the magnetic contribution $C_{\mathrm{mag}}=C_{p}-C_{\mathrm{ph}}$ to the specific heat after subtraction of the phonon contribution. By integrating the magnetic specific heat $C_{\text {mag }}$, we can obtain the magnetic entropy $S_{\mathrm{mag}}=\int_{0}^{T} \frac{C_{\mathrm{mag}}}{T} d T$ associated with the CEF ground state, which orders. $S_{\text {mag }}$ shows a plateau at about $R \ln 2$ corresponding to a doublet ground state.

To analyze the Stark splitting of our degenerate ground state due to the crystalline electric field (CEF) we search for solutions of the perturbation Hamiltonian for an eightfold cubic symmetry. For $f$-electron configurations, terms up to 
the sixth order are sufficient [38]:

$$
\mathcal{H}_{\mathrm{CEF}}=B_{4}\left(O_{4}^{0}+5 O_{4}^{4}\right)+B_{6}\left(O_{6}^{0}+21 O_{6}^{4}\right) .
$$

Here, the $O_{n}^{m}$ are the Stevens equivalent operators and the $B_{n}$ are the CEF amplitudes describing the admixture between the different states $\left| \pm \frac{9}{2}\right\rangle, \ldots,\left| \pm \frac{1}{2}\right\rangle$ of the $J$ multiplet.

To determine the ratio between fourth- and sixth-order terms, we follow the procedure laid out in Ref. [38], and substitute $O_{4}=O_{4}^{0}+5 O_{4}^{4}$ and $O_{6}=O_{6}^{0}-21 O_{6}^{4}$. Thus, we can rewrite Eq. (4) as

$$
\mathcal{H}_{\mathrm{CEF}}=W\left[x \frac{O_{4}}{F(4)}+(1-|x|) \frac{O_{6}}{F(6)}\right],
$$

where $B_{4} F(4)=W x$, and $B_{6} F(6)=W(1-|x|)$ for $-1<$ $x<+1$. This allows us to fit to the magnetic part of the specific heat $S_{\mathrm{mag}}$ for different values of $x$ and $W$ (see Fig. 2 in the Supplemental Material) in terms of a Schottky anomaly:

$$
\begin{aligned}
C_{\mathrm{CEF}}= & \frac{R}{T^{2}}\left[\frac{4 \Delta_{1}^{2} e^{-\frac{\Delta_{1}}{T}}+4 \Delta_{2}^{2} e^{-\frac{\Delta_{2}}{T}}}{2+4 e^{-\frac{\Delta_{1}}{T}}+4 e^{-\frac{\Delta_{2}}{T}}}\right. \\
& \left.-\left(\frac{4 \Delta_{1} e^{-\frac{\Delta_{1}}{T}}+4 \Delta_{2} e^{-\frac{\Delta_{2}}{T}}}{2+4 e^{-\frac{\Delta_{1}}{T}}+4 e^{-\frac{\Delta_{2}}{T}}}\right)^{2}\right] .
\end{aligned}
$$

For $\mathrm{Nd}^{3+}$ with a $J=9 / 2$, the 10 -fold degenerate ground state is lifted into a doublet $\Gamma_{6}$ as the ground state and the two quadruplets $\Gamma_{8}^{(1)}$ and $\Gamma_{8}^{(2)}$, which are separated by an energy gap of $\Delta_{1}$ and $\Delta_{2}$, respectively. We obtain a best fit shown as the solid line in Fig. 5 for $\Delta_{1}=29 \mathrm{~K}$ and $\Delta_{2}=72 \mathrm{~K}$. This allows two solutions, one with $x=-0.9650$ and $W / k_{B}=1.14 \mathrm{~K}$, and the other with $x=0.140$ and $W / k_{B}=0.774 \mathrm{~K}$.

Knowing the values of $x$ and $W$ allows us to calculate the expected magnetic moment of the $\Gamma_{6}$ doublet. For both solutions, this calculation yields a theoretical value of $1.83 \mu_{\mathrm{B}}$ for the ordered moment, which is close to the 1.78(9) $\mu_{\mathrm{B}}$ obtained from neutron diffraction.

\section{CONCLUSIONS}

We determined the magnetic structure of the semimetal $\mathrm{NdBiPt}$, which crystallizes in a half-Heusler structure. Below the Néel temperature $T_{\mathrm{N}}$ of $2.18 \mathrm{~K}$ we find an up-down structure of ferromagnetically aligned planes, in which the spin of the Nd points along the [001] direction, which alternate along the propagation vector $\tau=(100)$. This type-I structure is common for crystals belonging to the space group $F \overline{4} 3 \mathrm{~m}$. This opens the question of why in GdBiPt [23,24], YbBiPt [39], and vanadium-doped $\mathrm{CuMnSb}[40,41]$, the propagation vector of the antiferromagnetic structure (AFM) points along [111]. However, the magnetic structure we found in NdBiPt excludes this material from being a candidate for the proposed new class of antiferromagnetic topological insulators (AFTIs) [8]. In NdBiPt, the ground state is the $\Gamma_{6} \mathrm{CEF}$ doublet which orders, and we find an ordered moment of 1.78(9) $\mu_{\mathrm{B}}$.

\section{ACKNOWLEDGMENTS}

We thank O. Stockert from the MPI-CPFS in Dresden, Germany, for the discussion on double scattering. We also acknowledge the help from Oksana Zaharko from the PSI in Villigen, Switzerland, for her help with the FULLPROF refinement. The research at UdeM received support from the Natural Sciences and Engineering Research Council of Canada (Canada), Fonds Québécois de la Recherche sur la Nature et les Technologies (Québec), and the Canada Research Chair Foundation. Part of this work was supported by HLD at HZDR, a member of the European Magnetic Field Laboratory (EMFL).
[1] B. A. Bernevig, T. L. Hughes, and S.-C. Zhang, Science (New York, NY) 314, 1757 (2006).

[2] M. Konig, S. Wiedmann, C. Brune, A. Roth, H. Buhmann, L. W. Molenkamp, X.-L. Qi, and S.-C. Zhang, Science 318, 766 (2007).

[3] J. E. Moore, Nature 464, 194 (2010).

[4] M. Hasan and C. Kane, Rev. Mod. Phys. 82, 3045 (2010).

[5] D. Xiao, Y. Yao, W. Feng, J. Wen, W. Zhu, X.-Q. Chen, G. M. Stocks, and Z. Zhang, Phys. Rev. Lett. 105, 096404 (2010).

[6] D. Hsieh, Y. Xia, L. Wray, D. Qian, A. Pal, J. H. Dil, J. Osterwalder, F. Meier, G. Bihlmayer, C. L. Kane, Y. S. Hor, R. J. Cava, and M. Z. Hasan, Science 323, 919 (2009).

[7] H.-J. Zhang, C.-X. Liu, X.-L. Qi, X.-Y. Deng, X. Dai, S.-C. Zhang, and Z. Fang, Phys. Rev. B 80, 085307 (2009).

[8] R. S. K. Mong, A. M. Essin, and J. E. Moore, Phys. Rev. B 81, 245209 (2010).

[9] S. Chadov, X. Qi, J. Kübler, G. H. Fecher, C. Felser, and S. C. Zhang, Nat. Mater. 9, 541 (2010).

[10] W. Al-Sawai, H. Lin, R. S. Markiewicz, L. A. Wray, Y. Xia, S.-Y. Xu, M. Z. Hasan, and A. Bansil, Phys. Rev. B 82, 125208 (2010).
[11] C. Li, J. S. Lian, and Q. Jiang, Phys. Rev. B 83, 235125 (2011).

[12] P. C. Canfield, J. D. Thompson, W. P. Beyermann, A. Lacerda, M. F. Hundley, E. Peterson, Z. Fisk, and H. R. Ott, J. Appl. Phys. 70, 5800 (1991).

[13] Z. Fisk, P. C. Canfield, W. P. Beyermann, J. D. Thompson, M. F. Hundley, H. R. Ott, E. Felder, M. B. Maple, M. A. Lopez de la Torre, P. Visani, and C. L. Seaman, Phys. Rev. Lett. 67, 3310 (1991).

[14] G. Goll, J. Hagel, H. v. Löhneysen, T. Pietrus, S. Wanka, J. Wosnitza, G. Zwicknagl, T. Yoshino, T. Takabatake, and A. G. M. Jansen, Europhys. Lett. 57, 233 (2002).

[15] F. F. Tafti, T. Fujii, A. Juneau-Fecteau, S. René de Cotret, N. Doiron-Leyraud, A. Asamitsu, and L. Taillefer, Phys. Rev. B 87, 184504 (2013).

[16] N. P. Butch, P. Syers, K. Kirshenbaum, A. P. Hope, and J. Paglione, Phys. Rev. B 84, 220504 (2011).

[17] T. V. Bay, T. Naka, Y. K. Huang, and A. de Visser, Phys. Rev. B 86, 064515 (2012).

[18] T. Bay, M. Jackson, C. Paulsen, C. Baines, A. Amato, T. Orvis, M. Aronson, Y. Huang, and A. de Visser, Solid State Commun. 183, 13 (2014). 
[19] N. Kozlova, J. Hagel, M. Doerr, J. Wosnitza, D. Eckert, K.-H. Müller, L. Schultz, I. Opahle, S. Elgazzar, M. Richter, G. Goll, H. v. Löhneysen, G. Zwicknagl, T. Yoshino, and T. Takabatake, Phys. Rev. Lett. 95, 086403 (2005).

[20] K. Gofryk, D. Kaczorowski, T. Plackowski, J. Mucha, A. LeitheJasper, W. Schnelle, and Y. Grin, Phys. Rev. B 75, 224426 (2007).

[21] G. Xu, W. Wang, X. Zhang, Y. Du, E. Liu, S. Wang, G. Wu, Z. Liu, and X. X. Zhang, Sci. Rep. 4, 5709 (2014).

[22] C. Liu, Y. Lee, T. Kondo, E. D. Mun, M. Caudle, B. N. Harmon, S. L. Budko, P. C. Canfield, and A. Kaminski, Phys. Rev. B 83, 205133 (2011).

[23] A. Kreyssig, M. G. Kim, J. W. Kim, D. K. Pratt, S. M. Sauerbrei, S. D. March, G. R. Tesdall, S. L. Bud'ko, P. C. Canfield, R. J. McQueeney, and A. I. Goldman, Phys. Rev. B 84, 220408 (2011).

[24] R. A. Müller, N. R. Lee-Hone, L. Lapointe, D. H. Ryan, T. Pereg-Barnea, A. D. Bianchi, Y. Mozharivskyj, and R. Flacau, Phys. Rev. B 90, 041109 (2014).

[25] APEX2 and SAINT, Version 7.68A (Bruker AXS Inc., Madison, WI, 2009).

[26] G. M. Sheldrick, SADABS, Version 2008 (Bruker AXS Inc., Madison, WI, 2008).

[27] G. M. Sheldrick, Acta Crystallogr. Sect. A 64, 112 (2008).

[28] See Supplemental Material at http://link.aps.org/supplemental/ 10.1103/PhysRevB.92.184432 for an image of the two racemic crystal structures of GdBiPt, tables of the refinement parameters of our single crystal $\mathrm{x}$ ray, as well as the neutron data, and a figure showing the CEF level scheme for $\mathrm{a} \mathrm{Gd}^{+3}$ in a cubic environment.

[29] J. Wosnitza, G. Goll, A. D. Bianchi, B. Bergk, N. Kozlova, I. Opahle, S. Elgazzar, M. Richter, O. Stockert, H. v. Löhneysen, T. Yoshino, and T. Takabatake, New J. Phys. 8, 174 (2006).

[30] D. T. Morelli, P. C. Canfield, and P. Drymiotis, Phys. Rev. B 53, 12896 (1996).

[31] M. Fisher and J. Langer, Phys. Rev. Lett. 20, 665 (1968).

[32] T. Yildirim, A. B. Harris, and E. F. Shender, Phys. Rev. B 58, 3144 (1998).

[33] J. Rossat-Mignod, in Methods in Experimental Physics (Academic, New York, 1987) Chap. 19, pp. 69-157.

[34] J. Rodriguez-Carvajal, Physica B 192, 55 (1993).

[35] G. Shirane, S. M. Shapiro, and J. M. Tranquada, Neutron Scattering with a Triple Axis Spectrometer (Cambridge University, Cambridge, England, 2002).

[36] A. Pelissetto and E. Vicari, Phys. Rep. 368, 549 (2002).

[37] G. Goll, O. Stockert, M. Prager, T. Yoshino, and T. Takabatake, J. Magn. Magn. Mater. 310, 1773 (2007).

[38] K. Lea, M. Leask, and W. Wolf, J. Phys. Chem. Solids 23, 1381 (1962).

[39] B. G. Ueland, A. Kreyssig, K. Prokeš, J. W. Lynn, L. W. Harriger, D. K. Pratt, D. K. Singh, T. W. Heitmann, S. Sauerbrei, S. M. Saunders, E. D. Mun, S. L. Bud'ko, R. J. McQueeney, P. C. Canfield, and A. I. Goldman, Phys. Rev. B 89, 180403 (2014).

[40] R. Forster, G. Johnston, and D. Wheeler, J. Phys. Chem. Solids 29, 855 (1968).

[41] M. Halder, S. M. Yusuf, A. Kumar, A. K. Nigam, and L. Keller, Phys. Rev. B 84, 094435 (2011). 